Cultures \& Conflits

08 | hiver 1992

Les conflits après la bipolarité

\title{
Influences extérieures et identités au maghreb : le jeu du transnational
}

\section{Rémy Leveau}

\section{(2) OpenEdition \\ 1 Journals}

\section{Édition électronique}

URL : http://journals.openedition.org/conflits/527

DOI : $10.4000 /$ conflits. 527

ISSN : $1777-5345$

Éditeur :

CCLS - Centre d'études sur les conflits lilberté et sécurité, L'Harmattan

Édition imprimée

Date de publication : 6 décembre 1992

ISSN : 1157-996X

\section{Référence électronique}

Rémy Leveau, «Influences extérieures et identités au maghreb : le jeu du transnational », Cultures \& Conflits [En ligne], 08 | hiver 1992, mis en ligne le 27 janvier 2003, consulté le 30 mars 2021. URL:

http://journals.openedition.org/conflits/527 ; DOI : https://doi.org/10.4000/conflits.527

Ce document a été généré automatiquement le 30 mars 2021.

Creative Commons License 


\title{
Influences extérieures et identités au maghreb : le jeu du transnational
}

\author{
Rémy Leveau
}

1 Si les affrontements entre les islamistes et le pouvoir au Maghreb se déroulent encore dans le cadre des Etats ils contribuent à le remettre en cause ${ }^{1}$. En effet ces opposants occupent une part dominante de l'imaginaire social et essaient de transformer en utilisant les failles du système cette emprise en pouvoir politique. Les Etats eux fixent encore les règles du jeu et font appel aux centurions lorsque le contrôle de la situation leur échappe. Mais une vue sommaire consisterait à présenter cette rivalité comme si elle se déroulait dans un champ clos.

2 Pour apprécier les ressources et les chances des adversaires, il convient de réintroduire les variables extérieures. Celles-ci interviennent sous la forme d'influences multiples, elles se contrarient et s'insèrent dans un débat interne intense. Curieusement les événements du Maghreb ont aujourd'hui peu d'écho à l'extérieur, par comparaison avec le passé. Certes le FIS ou la monarchie marocaine peuvent avoir une visibilité dépassant le cadre national et les perceptions des changements en cours au Maghreb ont une audience, d'autant plus forte qu'elle est fréquemment traduite en termes catastrophiques par les medias dans les pays de l'Europe latine. Mais si l'on dépasse ce voisinage complice, l'intérêt suscité par les courants religieux du Moyen-Orient, le conflit israélo-arabe ou les problèmes des monarchies pétrolières ont une audience internationale beaucoup plus large que les crises maghrébines. La crise du Golfe et l'effondrement des pays de l'Est sur la scène internationale contribuent encore plus à marginaliser le Maghreb à l'échelle mondiale. Face à cette évolution, il ne faut cependant pas négliger les liens qui se sont tissés, au niveau des sociétés entre l'Europe et le Maghreb à partir d'une émigration installée, qui conserve des attaches multiples et un intérêt pour le devenir des pays d'origine. Cette émigration peut rétablir un courant d'échanges et de valeurs communes qui fonderont le dialogue et justifieront les aides susceptibles de réduire les risques d'évolutions catastrophiques. Elle pourrait aussi à l'inverse transformer l'affrontement en cours aujourd'hui au Maghreb en une 
nouvelle forme de confrontation transnationale mobilisant des solidarités extérieures, construisant une "menace du Sud" islamiste en remplacement du "péril rouge"2.

Si l'Europe et le Maghreb veulent éviter d'en arriver à ces enchaînements d'absurdités construites à partir d'imaginaires fortement antagonistes, elles doivent reconstituer des espaces de valeurs et de solidarités communes, en commençant peut-être par réintégrer dans leurs perceptions d'aujourd'hui ce qui a pu exister dans le passé. Des réseaux d'échanges où circulaient les hommes, les valeurs, les marchandises ont en effet fonctionné dans un contexte essentiellement bilatéral entre le Maghreb et la France, pendant la période coloniale. La notion d'appartenance à l'"Empire" français comportait, dans un cadre il est vrai inégalitaire, des éléments qui pouvaient justifier une sorte de pacte où les élites colonisées ont pu trouver avantage pour un temps. Cette situation a entraîné la participation acceptée des Maghrébins à la défense de la France dans les deux guerres mondiales. En revanche, le non-respect de ce pacte par la France détermine la protestation nationaliste du capitaine AbdelKader en 1925, ou les émeutes de Sétif du 8 mai 1945. Au lendemain des indépendances, la croyance en des valeurs communes anime la mise en oeuvre de la "coopération" qui, à bien des égards et dans une situation d'ambiguïté partagée, fleurit sur les décombres de l'Empire. Pour les Français, on pouvait y voir la préservation de l'influence sans la souveraineté par le biais de la langue et de la construction d'Etats sur le modèle jacobin. De l'ex-Empire on conservait l'école de Jules Ferry dans un autre registre. Du côté maghrébin, c'est le volet modernité qui l'emportait avec un raisonnement qui pouvait, sur le plan interne, présenter la coopération comme les "réparations" de la colonisation, et donc justifier l'acceptation d'une aide qui comportait une part de dépendance.

4 Ce système ne pouvait survivre que dans une logique de transition. Pour se guérir des regrets de l'Empire, la France s'est lancée dans la construction de l'Europe, la force de frappe et quelques autres grands projets. Les pays du Maghreb ont suivi des voies divergentes, comportant leur part de projets économiques et politiques ambitieux, construits notamment dans le cas de l'Algérie sur un discours de politique étrangère fortement opposé aux préoccupations françaises et européennes. Le point de divergence se situe certainement dans les années soixante-dix, lorsque la France doit choisir entre une coopération qu'elle veut entreprendre avec les nouveaux pays industriels et le maintien de liens avec le Maghreb qui tendent à absorber la totalité de ses ressources. La décision de faire payer progressivement $80 \%$ du coût réel des coopérants conduira à l'arabisation de l'enseignement secondaire en quelques années, la facilité de recrutement des coopérants français à bon marché ayant servi jusque là d'alibi pour maintenir l'enseignement des disciplines scientifiques en langue étrangère. Avec le départ progressif des coopérants (20 000 personnes environ en 1975), c'est un tissu de rapports humains, de connaissance mutuelle et de contacts personnels au niveau de la classe moyenne et des élites qui se défait. En même temps, les besoins de modernisation accrus par la croissance démographique et par l'urbanisation des populations deviennent de plus en plus difficiles à satisfaire et la coopération ne peut en couvrir qu'une part marginale.

5 Parallèlement un autre milieu humain porteur d'échanges se recrée sur la rive Nord de la Méditerranée avec l'installation de l'immigration maghrébine, mais sans élites propres. Il faudra quelques temps pour que la greffe porte ses fruits et pour qu'une prise de conscience du changement ait lieu, en ce qui concerne entre autres l'inversion des flux d'enseignants des disciplines scientifiques ${ }^{3}$. 
Dans la transition, les anciennes valeurs communes inavouées finissent par disparaître ou tomber en désuétude. Les problèmes d'identité des pays du Maghreb font que l'arabisation, la récupération ou l'interdiction de fréquentation des établissements français sont perçus comme des volontés de rupture - alors que les querelles portant sur le "Hizb Fransa" sont d'abord des conflits des Maghrébins avec eux-mêmes. La perception dramatisée de la rupture à lieu à l'occasion de la crise du Golfe "On a compté pour moins que rien" dit-on du côté maghrébin, "Pourquoi sont-ils si violents et si irrationnels" dit-on en face. L'intensité de la crise disparaitt vite, mais les interrogations demeurent. Lorsque les Maghrébins se posent le problème de la sortie des relations bilatérales et d'une coopération avec un espace européen unifié, ils ont le sentiment désagréable que l'Europe n'a pas besoin d'eux alors qu'ils ont besoin de l'Europe.

7 Le commerce extérieur de la CEE avec le Maghreb représente $1 \%$ de ses échanges alors qu'il compte pour près de $70 \%$ dans l'autre sens. L'Europe ne veut plus des Maghrébins comme travailleurs. D'autres pays sont prêts à se substituer à eux comme exportateurs de matières premières. Leurs cuirs, leurs textiles sont mis en concurrence avec des produits équivalents d'Europe de l'Est ou des pays asiatiques. Leurs productions agricoles sont refoulées au profit de celles de l'Europe du Sud. Là où une demande existe, dans le domaine de la pêche par exemple, ils ont le sentiment d'être l'objet d'un pillage et le tourisme, qui reste un des secteurs importants des échanges pour la Tunisie et le Maroc pose des problèmes d'identité qu'expriment les récriminations et parfois les violences des islamistes.

Depuis l'effondrement des pays de l'ex-bloc soviétique, les élites maghrébines savent qu'elles n'ont d'autre recours qu'une entente avec l'Europe. Un accord du style de celui qui instaure en 1992 une zone de libre-échange entre le Mexique, les USA et le Canada les tente, mais elles ont le sentiment d'un partenariat inégal et moralement impossible. D'un autre côté, les pays de l'Europe du Nord ne voient pas de raisons de se lier par des accords particuliers avec le Maghreb plutôt qu'avec les pays de l'Est, la Turquie ou l'Egypte, pour se limiter à l'environnement immédiat de la Communauté. Dans l'état actuel des relations, certains pays de l'Est ou la Turquie ont sans doute plus de chances d'entrer dans une association avec l'Europe que le Maghreb.

9 La chance particulière du Maghreb (qui vaut aussi dans un autre contexte pour la Turquie) est de jouer sur les solidarités qui se sont bâties au niveau des sociétés civiles à partir des immigrations installées dans l'Europe du Nord (France, Allemagne, Belgique, Hollande). Globalement, un groupe de 3,5 à 4 millions de personnes d'origine maghrébine est installé à demeure ${ }^{4}$. Quels que soient aujourd'hui les rapports instrumentaux entretenus avec les nationalités des pays d'origine ou des pays d'accueil. Elles aspirent à disposer des deux nationalités et, demain, d'une citoyenneté européenne en plus. Ces populations sont rejetées sur ce plan comme sur beaucoup d'autres, tantôt du côté des pays d'origine, tantôt vers l'Europe. Néanmoins, elles resteront là où elles sont et faciliteront le plus possible le passage en Europe de leurs proches.

10 Les communautés maghrébines en France tendent à constituer un espace de liberté, politique ou économique, qui ne peut exister au Maghreb du fait des systèmes de contrôle de l'information et des pénuries diverses. Les dirigeants maghrébins cherchent donc à maîtriser, légalement ou non, cette marge d'autonomie où l'on voit bientôt une forme d'opposition politique. Ils se plaignent souvent d'un manque de coopération des autorités européennes dans ce domaine. Ils réussiront quelquefois à 
limiter l'exercice du droit de manifestation dont disposent leurs ressortissants, et même à établir avec les autorités policières françaises des formes de collaboration visant à surveiller leurs adversaires, à limiter la liberté de la presse, et à faire expulser les éléments les plus engagés. Cela n'aboutira, comme au temps des conflits d'indépendance, qu'à transférer en Allemagne, en Suisse et en Angleterre les éléments les plus engagés, notamment parmi les islamistes.

11 Mais, avec la crise du Golfe, les pays du Maghreb vont s'apercevoir avec inquiétude que l'Europe se désintéresse globalement de leur sort, l'Egypte comptant plus qu'eux dans le jeu régional qui se dessine. Les relations bilatérales sur lesquelles s'appuyaient leurs rapports avec les pays européens et avec la France en particulier se révèlent une illusion.

12 L'Europe devient une figure menaçante qui bombarde la rive Sud de la Méditerranée d'images hostiles et humiliantes, et traite à un niveau collectif et anonyme les questions qui étaient jusqu'alors négociées d'Etat à Etat. Partant de leur propre expérience du champ multilatéral maghrébin, de leurs souvenirs et de leur nostalgie de la politique arabe du général De Gaulle, les élites maghrébines ne veulent pas croire que la construction européenne puisse se faire au prix d'abandons de souveraineté dont les conséquences marginalisent leurs pays dans leurs rapports avec l'Europe.

13 Sous cet angle, le partage de l'aide européenne avec les pays de l'Est apparaît comme une menace plus pesante que la régulation d'un champ migratoire soumis à de nouvelles influences. Jusqu'alors, les pays du Maghreb s'accommodaient bien de la fiction d'un arrêt de l'immigration en 1974 qui satisfaisait leur dignité, et de la réalité d'une circulation souple, favorisée par des canaux suffisamment ouverts pour assurer une soupape de sûreté et une part de rêve aux jeunes sans emploi. L'imposition de visas aux touristes, sous le prétexte de la lutte contre le terrorisme en 1986, avait constitué un premier accroc au fonctionnement d'un système aspirant avant tout à la liberté de circulation des personnes et ne souhaitant pas s'associer sans contrepartie à des contrôles susceptibles de compliquer les relations avec sa population. Peu importe que l'Europe constitue, à partir de 1985, un réseau de gestion commune des visas sur une base bilatérale (accords de Schengen) qui annonce la mise en place à terme d'une politique de contrôle des flux migratoires. Il reste pendant longtemps assez de souplesse - grâce au passage par l'Italie et l'Espagne - pour que l'entrée en France ou en Belgique ne soit pas totalement bloquée. Le Maghreb ne se sent pas plus concerné par ces décisions que par la politique communautaire relative aux réfugiés. Les visas de touristes ou d'étudiants, le regroupement familial, la réintégration dans la nationalité française, la souplesse des procédures de naturalisation sont des mécanismes qui fonctionnent dans la discrétion et suffisent à maintenir une certaine modulation des flux, malgré l'impossibilité de circuler librement. En plus des contraintes légales, le véritable obstacle à une liberté de circulation provient de la dépréciation des monnaies maghrébines qui rend prohibitif un séjour en Europe pour ceux qui n'appartiennent pas au monde des affaires, de la contrebande, ou à la haute nomenklatura.

Par ailleurs, les contraintes des nouvelles règles européennes ne sont pas directement perçues dans la mesure où l'imposition des visas, en 1986, s'accompagne du maintien et même d'un accroissement des échanges entre les élites au pouvoir à des fins sécuritaires. Les responsables maghrébins recherchent et obtiennent souvent une collaboration leur permettant de contrôler leurs opposants à l'extérieur. En retour ils collaborent à la lutte contre le terrorisme et les prises d'otages au Moyen-Orient. Un 
esprit de solidarité contre les mouvements islamiques crée un lien de complicité comparable à la lutte contre le gauchisme dans d'autres circonstances. Ces divers phénomènes convergents relativisent la perception de la construction institutionnelle qui se met en place. Les accords de Schengen apparaissent comme un subterfuge inavouable utilisé pour replacer dans un cadre multilatéral contraignant le statu quo antérieur.

Dans la phase transitoire de Schengen, les contraintes du contrôle des flux de personnes vont être adoucies par les facilités offertes par l'émigration installée: transferts financiers, certificats d'hébergement. Une gestion raisonnée des circuits d'échanges familiaux est utilisée pour les études ou les vacances des enfants; un "marché" des mariages et des soins médicaux se met en place avec sa part de rétributions matérielles et symboliques. Cette interaction produit un système de mobilité transnationale et aboutit à une double insertion de l'immigration qui fonctionne au niveau des sociétés quels que soient les conflits et les exclusions qui l'accompagnent. Globalement, ce processus renforce le poids de l'émigration installée en Europe dans les affaires du pays d'origine, et crée une sorte de groupe de pression pesant en faveur d'un jeu politique pluraliste, de valeurs libérales notamment sur le plan économique tout en renforçant la demande de démocratisation.

16 La crise de 1990-1991, liée à l'affaire du Golfe, remet en cause les ajustements intervenus tant au niveau des élites gouvernantes que des sociétés. Elle réduit considérablement la signification des liens bilatéraux et place l'immigration maghrébine dans une situation délicate et précaire. Sa solidarité avec les opinions publiques des pays du Maghreb ne pourra aller au-delà d'un sentiment douloureux d'impuissance, mais elle ne contestera à aucun moment le droit du chef de l'Etat français d'engager la France dans une guerre qui l'oppose à un pays arabe. Prudente et malheureuse, l'immigration privilégie son insertion et se sépare sans le dire des manifestations émotionnelles des foules urbaines et des élites maghrébines ${ }^{5}$. Exprimé avec discrétion, ce décalage n'en sera pas moins ressenti de l'autre côté de la Méditerranée comme un nouvel abandon.

17 Cette dissociation, vécue là-bas comme un reniement et ici comme une éternelle suspicion, coïncide avec l'adhésion en novembre 1990 de l'Italie, puis en mai 1991 de l'Espagne, au système de Schengen. Par anticipation, une politique commune des visas est mise en place avec le Maghreb. Le sentiment de coupure et d'isolement, mêlé de fascination et de frustration, s'amplifie considérablement à un moment où les villes maghrébines ont l'impression d'avoir tous les jours rendez-vous avec l'Europe au journal télévisé de 20 heures. Mais la décomposition de l'Etat et des sociétés maghrébines n'atteint pas le niveau de certains pays de l'Est, et l'image de l'Europe exerce toujours un attrait mêlé de rejet. Toutefois, avec l'imposition généralisée des visas, le sentiment d'exclusion domine, associé à celui de ne pouvoir résister à l'intrusion de cette même société qui vous repousse. L'Europe est perçue comme une forteresse dont la conquête commence avec la queue pour les visas auprès des consulats européens.

Malgré ces entraves, les flux de population restent considérables. Les consulats de France dans les différents pays du Maghreb ont délivré près de 900000 visas en 1991. Plus d'un million de Maghrébins transitent chaque année durant l'été par l'Espagne. Le téléphone automatique, quelques heures d'avion, suffisent pour approcher un univers dont l'accès est fermé à celui qui ne dispose ni d'influence ni d'argent. La situation est 
d'autant plus insupportable que la France reste pour les élites maghrébines, productrice de normes et de valeurs, même si le discours courant à son égard est avant tout celui de la contestation et de la récrimination.

Dans ce contexte, les politiques volontaristes ont peu d'emprise. Cela vaut autant pour les systèmes de contrôle et de visas des pays européens que pour les politiques autoritaires des Etats maghrébins dont la gestion maladroite ne peut qu'accroître les perceptions hostiles. La libre circulation est impossible, créatrice de fantasmes dans les sociétés européennes et destructrice du tissu social des sociétés maghrébines, notamment du fait de l'exode des élites. Les contrôles absolus créant des effets pervers, les choix ne peuvent s'inspirer que du principe du moindre mal et tolérer une part de transgression qui rendra acceptable, tant au niveau des sociétés maghrébines que des immigrations installées, les nouvelles contraintes déterminées par l'espace européen unifié. Le maintien d'un certain niveau de solidarités transnationales passant par les sociétés civiles pourra peut-être se traduire des deux côtés par une politique plus réaliste des Etats.

Il serait sans doute préférable d'aboutir à une stratégie communautaire mieux affichée du côté européen pour sortir du psychodrame des relations bilatérales. Une collaboration avec les Etats maghrébins visant à renforcer leur rôle, sans prétendre pour autant exercer de contrôle sur le choix des partenaires en récusant à l'avance les islamistes, peut éviter les dérives dangereuses. Mais une conditionnalité de l'aide en termes de respect des droits de l'Homme peut contenir un exode de la classe moyenne qui minerait les Etats et entraînerait en Europe des réactions politiques difficiles à gérer. La contrepartie à une collaboration des Etats maghrébins aux contraintes rendues nécessaires par le contrôle des flux de population se situera en termes d'aménagement de la dette et d'aide économique. La création d'un espace d'interaction économique, de convertibilité des monnaies et de développement intégré au niveau des pays de l'UMA pourrait offrir aux jeunes Maghrébins un autre espoir que la démarche, nourrie d'illusions et porteuse d'un sentiment de reniement, qui les conduit par tous les moyens de l'autre côté de la Méditerranée et fait craindre à l'Europe, et en premier lieu à l'Espagne, d'être soumise à des pressions difficilement contrôlables de type albanais débarquant en Italie.

21 La prise en compte de cette réalité suppose, de part et d'autre, l'acceptation de valeurs communes. Le droit au séjour durable ne peut se réaliser sans une certaine intégration aux valeurs du groupe majoritaire. En retour les droits et les cultures des minorités doivent être reconnus suivant des modalités négociées. Concrètement, l'intégration de l'immigration maghrébine suppose que l'on reconnaisse une place à l'islam dans la construction de l'Europe, c'est-à-dire dans la définition des normes et des valeurs communes. Si ce but est atteint, les changements doctrinaux qui se produiraient dans l'espace européen ne manqueront pas d'influencer le Maghreb. L'extension du pluralisme à l'islam peut recréer les bases à partir desquelles de nouvelles solidarités peuvent se reconstruire, associant les sociétés et les Etats, l'immigration jouant un rôle d'intermédiaire dans un processus de double insertion positive dans les deux systèmes.

Si un tel cadre s'établit, il devrait permettre la libre circulation des idées et progressivement des personnes et des marchandises. De grands projets à valeur symbolique, comme le gazoduc et la liaison continue à travers le détroit de Gibraltar, devraient y trouver leur place. Dans cette perspective, l'aide au développement du Maghreb pourrait, sous des formes diverses qui concernent aussi bien des 
délocalisations de fabrication (textile, automobile) que des contingents d'immigration, des prêts et des dons, redonnerait aux Etats et aux populations l'espoir d'un changement. Cet espoir pourrait aussi, sur le plan intérieur maghrébin, faciliter des compromis et des transitions qui ne se feraient, autrement, que par l'épuisement des adversaires.

Mais un tel partenariat qui se déroulerait nécessairement pour l'essentiel avec les Etats suppose que l'on puisse parler de toutes les questions conflictuelles et non pas seulement de celles qui peuvent se traduire en termes de consensus anodin ou de complicité entre les élites. Les politiques démographiques, sans lesquelles les plans d'aide à long terme n'ont aucun sens, devraient faire partie des réflexions communes tant en termes d'aide que de régulation des flux migratoires. Les problèmes d'armement, de dénucléarisation, et de renoncement de part et d'autre à la violence intermédiatrice font aussi partie des fondements d'une nouvelle politique de bon voisinage, quelles que soient les formes, institutionnelles ou non, de ces échanges qui concernent aussi les sociétés.

L'intérêt pour le Maghreb peut être de trouver dans ce type de construction un espace plus vaste sur le plan économique, culturel et humain pour résoudre les problèmes de transition économique et politique qui sont les siens. L'intérêt de l'Europe est de sortir d'une problématique de rapports internationaux à l'état brut où le plus puissant impose ses contraintes du moment et le plus faible joue, souvent de façon inorganisée, sur sa capacité de nuisance.

En se situant dans une logique de situation non gérée, on peut voir l'Europe imposant la projection de ses images, créant des tentations dévastatrices, imposant une obligation de visa au moment où elle cherche à obtenir un espace de libre circulation pour ses marchandises, ses touristes et ses bateaux de pêche, et donnant le moins d'aide possible en contrepartie des ouvertures négociées, à partir de l'idée que l'aide ne servira de toute façon à rien tant que les Etats n'auront pas atteint un niveau de stabilité qui permet un développement.

Du côté des dirigeants maghrébins, l'aide tend à être considérée comme un dû, comme une réparation du préjudice colonial ou des inégalités de la répartition de la rente pétrolière. Bilatérale ou internationale, elle ne doit comporter ni contrôles ni contraintes. C'est la contrepartie d'une injustice et si le développement tel qu'il est conçu à l'extérieur doit créer une situation d'infériorité permanente, d'inégalité à l'intérieur de la société, il n'est pas réellement souhaité. Les sociétés maghrébines se contenteront d'une aide qui prendra à la longue la forme d'une sorte de RMI international ou d'une pension alimentaire en faveur du prolétariat mondial pour qu'il se tienne tranquille.

27 En matière de mouvements de personnes, le Maghreb souhaite trouver en Europe une absence de réglementation, un "laissez-faire, laissez-passer" que certains dirigeants rationalisent par un discours d'émigration temporaire et d'allégeance perpétuelle des partants et de leurs descendances parce que, à l'intérieur, toute autre position est illégitime. Allant plus loin, les islamistes raisonneront en termes de retour des émigrés mais aussi d'enclave et de communauté si l'installation doit être durable. Dans la logique des imaginaires catastrophiques qui se développent à partir d'une réalité mal dominée, on pourrait passer de la situation d'émigration sauvage actuelle, où des pateras déposent leurs cargaisons d'illégaux sur les plages espagnoles, à un déferlement. 
28 La culture du haschish, dont les superficies dans le Rif ont dû être multipliées par dix depuis l'indépendance, peut encore s'étendre, se convertir en culture du pavot et installer ses laboratoires de transformation d'héroïne dans une situation de type colombien à la porte de l'Europe. L'industrie des faux passeports, la contrebande d'armes, les contrefaçons diverses peuvent se combiner, si les Etats s'affaiblissent, dans un contexte général dominé par des rapports de force, internes et externes, dont le Liban des milices donnait une idée.

29 Une évolution anarchique du Maghreb aboutirait à la destruction des Etats ou du moins à la limitation de leur rôle à une défense minimale des avantages acquis par les groupes au pouvoir s'appuyant sur la force dont ils disposent. Le champ social pourrait alors être occupé par des groupements d'intérêts ethniques ou communautaires, qui mettraient la main sur les ressources disponibles ou créeraient les moyens de faire fonctionner des communautés délinquantes, en marge de l'Etat et de l'ordre international, en ayant recours à n'importe quelles utopies justificatrices - religieuses ou politiques. On est certes très loin de cette situation caricaturale et les Etats maghrébins, en dépit des crises qu'ils connaissent, restent puissants, laissant au moins à l'Europe le temps de choisir entre le modèle mexicain et le modèle colombien pour bâtir ses relations avec le Maghreb.

Du côté maghrébin, la construction d'une identité islamique perçue par l'Europe comme un danger ou comme une provocation apparait comme la seule réponse possible face à la domination omniprésente du modèle occidental. Mais ce discours de sauvegarde sera nécessairement interprété comme une rupture tant avec les formes classiques de la modernité qu'avec toute tentative visant à rebâtir des solidarités nouvelles, y compris avec l'émigration maghrébine installée en Europe. Par contre, au niveau des populations, il sera ressenti comme une tentative de continuité culturelle soucieuse de maitriser la technique sans se laisser corrompre.

31 Le modèle mondial de l'univers technique apparaît acceptable s'il est détaché du système des valeurs auquel il est associé. L'islamisme apparaît aussi comme un mouvement social capable de transcender les terroirs et d'unifier des sociétés tentées par l'éclatement. Il reprend cette tâche là où le nationalisme du lendemain des indépendances l'avait laissée inachevée. Mais il le fait au prix de l'exclusion des élites modernisatrices qui ont assuré jusqu'ici la transformation de la société et le maintien de certaines solidarités avec un monde extérieur européen et occidental, et qu'ils accusent de cosmopolitisme. Ils répondent ainsi au rejet dont ils sont l'objet de la part de ces élites par une exclusion qui rappelle celle des juifs et des colons lors de l'effondrement de la construction coloniale. Pour asseoir leur révolte ils savent mobiliser les groupes sociaux laissés en dehors des tentatives réformatrices des Etats.

32 Mais les gouvernants en place ne sont pas décidés à se laisser submerger sans combattre. La fin de la guerre du Golfe a redonné aux appareils politiques le sentiment qu'une reprise en main était possible et nécessaire, s'ils ne voulaient pas se laisser déborder à l'occasion d'une nouvelle crise. Les Etats ne sont guère mieux placés que les islamistes pour obtenir de l'extérieur la création de solidarités nouvelles, si ce n'est en jouant sur la crainte que peuvent inspirer ces adversaires. En attendant, ils restent forts et la complicité des classes dirigeantes avec l'extérieur leur procure des soutiens non négligeables. Mais il faut passer de la complaisance entre les élites internationales à la construction de solidarités plus largement partagées, à la mesure des flux d'images, de marchandises ou de personnes qui transitent entre le Maghreb et l'Europe. Cela 
suppose qu'il soit possible d'intégrer dans la construction de ces solidarités des facteurs qui sont encore trop souvent considérés, de part et d'autre, comme appartenant à un domaine non négociable et non partagé avec l'étranger.

\section{NOTES}

1. Voir Bernard Botiveau : "De nouveaux modes de contestation dans le monde arabe" in Cultures \& Conflits : Emeutes urbaines le retour du politique, n 5, printemps 1992. 2. Voir Didier Bigo "l'idéologie de la menace du Sud" in Cultures \& Conflits $n^{\circ} 2$. 3. On peut aujourd'hui estimer à plus de 3000 le nombre des maîtres auxiliaires contractuels d'origine maghrébine enseignant pour combler les déficits dans les disciplines scientifiques au niveau des lycées et collèges - particulièrement sensibles dans les zones défavorisées. Il faudrait y ajouter dans ces mêmes disciplines quelques centaines de postes dans l'enseignement supérieur et la recherche scientifique.

4. Il dépasse en nombre celui des européens (environ 2 millions) vivant au Maghreb au moment des indépendances.

5. Les musulmans et la guerre du Golfe - Sondage IFOP - 4 février 1991.

\section{RÉSUMÉS}

Si la crise du Golfe et l'effondrement des pays de l'est, ont eu tendance à marginaliser le Maghreb et les crises qui le traverse sur la scène mondiale, ce dernier conserve cependant avec l'Europe des liens qu'il convient de ne pas négliger. Le continent européen exerce toujours un attrait mêlé de rejet, toutefois la construction européenne transforme de plus en plus le continent comme une forteresse dont la conquête commence avec la queue pour les visas. même si les flux de populations demeurent considérable. En outre les populations immigrés ou de deuxième génération pèse de plus en plus sur les rapports politiques entre les Etats européens et ceux du Maghreb. Le sentiment d'exclusion domine associé comme le remarque Rémy Leveau à celui de ne pouvoir résister à l'intrusion de cette même société qui vous repousse.

The Gulf crisis and the collapse of Eastern Europe have marginalised the Maghreb and its crises on the global scene. However, its links with Europe still place it in an important position. The European continent is at the same time a pole of attraction and of rejection. The construction of a united Europe has turned the continent into a fortress, the conquering of which starts at the queues for visas (although the migratory stream remains significant). Moreover, the immigrant population and its descendants play an important political role in the relations between European and Maghreb governments. A feeling of exclusion predominates, but it is realised that this society that excludes also exerts an irresistible attractive and intruding force. 
INDEX

Index géographique : Maghreb

Mots-clés : réseaux transnationaux, identité 